\title{
CD30-targeted oncolytic viruses as novel therapeutic approach against classical Hodgkin lymphoma
}

\author{
Julia D.S. Hanauer ${ }^{1}$, Benjamin Rengstl ${ }^{2,4}$, Dina Kleinlützum ${ }^{1}$, Johanna Reul ${ }^{1}$, Anett \\ Pfeiffer $^{1}$, Thorsten Friedel ${ }^{1}$, Irene C. Schneider ${ }^{1}$, Sebastian Newrzela ${ }^{2,4}$, Martin- \\ Leo Hansmann ${ }^{2}$, Christian J. Buchholz ${ }^{1}$ and Alexander Muik ${ }^{1,3}$ \\ ${ }^{1}$ Molecular Biotechnology and Gene Therapy, Paul-Ehrlich-Institut, Langen, Germany \\ ${ }^{2}$ Dr. Senckenberg Institute of Pathology, Goethe-University Frankfurt, Frankfurt am Main, Germany \\ ${ }^{3}$ Current address: BioNTech RNA Pharmaceuticals GmbH, 55131 Mainz, Germany \\ ${ }^{4}$ Current address: BioNTech Cell and Gene Therapies GmbH, 55131 Mainz, Germany
}

Correspondence to: Christian J. Buchholz, email: christian.buchholz@pei.de

Keywords: classical Hodgkin lymphoma; CD30; virotherapy; retargeting; VSV

Received: September 06, 2017 Accepted: December 03, $2017 \quad$ Published: January 12, 2018

Copyright: Hanauer et al. This is an open-access article distributed under the terms of the Creative Commons Attribution License 3.0 (CC BY 3.0), which permits unrestricted use, distribution, and reproduction in any medium, provided the original author and source are credited.

\section{ABSTRACT}

Classical Hodgkin lymphoma ( $\mathrm{CHL}$ ) is a hematopoietic malignancy with a characteristic cellular composition. The tumor mass is made up of infiltrated lymphocytes and other cells of hematologic origin but only very few neoplastic cells that are mainly identified by the diagnostic marker CD30. While most patients with early stage $\mathrm{cHL}$ can be cured by standard therapy, treatment options for relapsed or refractory cHL are still not sufficient, although immunotherapy-based approaches for the treatment of $\mathrm{cHL}$ patients have gained ground in the last decade. Here, we suggest a novel therapeutic concept based on oncolytic viruses selectively destroying the $\mathrm{CD} \mathrm{O}^{+}$-positive $\mathrm{CHL}$ tumor cells. Relying on a recently described CD30-specific scFv we have generated CD30-targeted measles virus (MV-CD30) and vesicular stomatitis virus (VSV-CD30). For VSV-CD30 the VSV glycoprotein G reading frame was replaced by those of the CD30-targeted MV glycoproteins. Both viruses were found to be highly selective for CD30-positive cells as demonstrated by infection of co-cultures of target and non-target cells as well as through blocking infection by soluble CD30. Notably, VSV-CD30 yielded much higher titers than MV-CD30 and resulted in a more rapid and efficient killing of cultivated cHL-derived cell lines. Mouse tumor models revealed that intratumorally, as well as systemically injected VSV-CD30, infected cHL xenografts and significantly slowed down tumor growth resulting in a substantially prolonged survival of tumor-bearing mice. Taken together, the data support further preclinical testing of VSV-CD30 as novel therapeutic agent for the treatment of cHL and other CD30+-positive malignancies.

\section{INTRODUCTION}

Classical Hodgkin lymphoma (cHL) is a malignant disease of the hematopoietic system and occurs with an incidence of 3-4 cases per 100.000 persons per year [1]. $\mathrm{cHL}$ is characterized by a unique histological pattern, as tumor cells of cHL are composed of Hodgkin and ReedSternberg (HRS) cells representing a mononucleated or multinucleated subtype, respectively. The most important immunological markers on these HRS cells are CD30 and
CD15. Importantly, cHL cells only account for $<1 \%$ of the tumor mass. The tumor cells are accompanied by a characteristic reactive infiltrate, mainly consisting of activated lymphocytes [2]. This implies that very few neoplastic cells recruit a great amount of lymphocytes and create a microenvironment favorable for tumor progression. Especially $\mathrm{CD}^{+} \mathrm{T}$ cells are involved in the so-called rosetting of tumor cells. Their presence correlates with poor progression-free and overall survival [3]. Current standard treatment for patients with early 
stage cHL includes multi-agent chemotherapy and localized radiotherapy [4]. Patients with chemosensitive relapse of cHL are treated by autologous hematopoietic stem cell transplantation (HSCT). This treatment results in long-term progression-free survival in approximately $50 \%$ of patients [5]. Therapeutic concepts resulting in a permanent antitumoral response in patients who failed autologous HSCT are therefore still needed. Recent concepts have started to focus on immunotherapy approaches. As targeting molecule, CD30 is in focus since it is minimally expressed on normal cells [6]. The most prominent example for a CD30-directed medicinal product is brentuximab vedotin, a CD30-specific monoclonal antibody coupled to a cytotoxic microtubule disrupting agent, which recently received marketing authorization [6]. Applied as salvage therapy for patients with relapsed cHL and with a $41 \%$ 5-year overall survival rate it has substantially improved the situation for these patients [7]. Further improvement can be expected from the PD1specific immune checkpoint inhibitor nivolumab, which was approved in 2016 for the treatment of patients with $\mathrm{cHL}$ that have relapsed or progressed after HSCT and brentuximab vedotin treatment [5]. However, with a $65 \%$ objective response rate there is still need for improvement and alternative concepts.

Oncolytic viruses combine the selective destruction of tumor cells with the induction of an antitumoral immune response [8]. They have so far been developed for many cancer entities and reached marketing authorization for the treatment of melanoma with the herpesvirus-based product Imlygic [9]. For cHL, however, virotherapy has so far not been considered. The low frequency of tumor cells in the affected tissue, as compared to other tumor entities, makes the use of conventional oncolytic viruses challenging. Additional layers of tumor cell targeting may in the case of cHL be instrumental to establish an effective virotherapy strategy. Cell type specific infection at the level of cell entry has been established for some enveloped oncolytic viruses such as measles virus (MV) or herpesvirus $[10,11]$. In case of MV, cell entry is mediated by the receptor attachment protein hemagglutinin $(\mathrm{H})$ and the membrane fusion protein F. Usage of the natural receptors CD46, signaling lymphocyte activation molecule (SLAM), and nectin-4 for cell entry can be redirected to a cell surface receptor of choice by introducing point mutations in $\mathrm{H}$ and fusion to a targeting ligand with high affinity for the selected surface marker. By this means a series of measles viruses have been generated each using a particular type of tumor surface marker as receptor $[12,13]$. All these viruses are highly selective for their target cells and replicate as efficiently as unmodified MV. Recently it was shown that vesicular stomatitis virus (VSV) can be receptortargeted by replacing its glycoprotein $\mathrm{G}$ gene against the glycoproteins of MV. Compared to MV, it replicates faster and can be produced at higher titers $[14,15]$.
In the present work, we generate the first examples of CD30-targeted oncolytic viruses. We compare the oncolytic activities of MV and VSV on cHL tumor cell lines in vitro and in vivo. VSV-CD30 was found to be especially promising for further preclinical studies, since it was active also upon systemic administration and in some mice even in a disseminated tumor mouse model.

\section{RESULTS}

\section{Generation of CD30-targeted oncolytic viruses}

With the aim of selectively destroying CD30 ${ }^{+}$-positive cHL-cells, both, CD30-targeted measles virus (MV-CD30) and vesicular stomatitis virus (VSVCD30), were generated. As CD30-specific binding domain the recently described scFv HRS3opt2\#2 was used [16]. Its coding sequence was fused to that of the MV hemagglutinin $(\mathrm{H})$ variant, which has been blinded for usage of the natural MV receptors CD46 and SLAM [17]. For MV-CD30 the unmodified MV-H gene was exchanged against the coding sequence of $\mathrm{H}_{\text {mut }}-\mathrm{CD} 30 \mathrm{scFv}$, whereas in case of VSV-CD30 the VSV glycoprotein G gene was replaced by the reading frames of the MV fusion protein (F) and $\mathrm{H}_{\text {mut }}-\mathrm{CD} 30 \mathrm{scFV}$ (Figure 1A). After rescue of the oncolytic viruses (OVs), they were propagated on Vero- $\alpha$ His cells, which are CD30negative but display a Hexa-His-tag (H6)-specific antibody which can be used as entry receptor by both viruses due to a C-terminal $\mathrm{H} 6$ fused to $\mathrm{H}_{\text {mut }}-\mathrm{CD} 30 \mathrm{scFv}$. As a control MV-CD30, VSV-MV, VSV-CD30 containing an unmodified $\mathrm{H}$ protein (without H6) was rescued as well.

To verify the molecular composition of the rescued viruses, Western blot analysis was performed. VSV-CD30 and VSV-MV contained the MV protein $\mathrm{F}$ and $\mathrm{H}$ along with the VSV proteins N, P and M (Figure 1B). The VSV $G$ protein was only detectable in stocks of VSV but not in the VSV-MV chimeric viruses. In correspondence to the fused $\mathrm{scFv}$ protein, the electrophoretic mobility of $\mathrm{H}_{\text {mut }}{ }^{-}$ CD30scFv was reduced when compared to $\mathrm{H}$. This was also the case for stocks of MV-CD30 which were analyzed along with MV stocks (Figure 1B). The incorporation of the CD30-scFv did not influence the replication of VSVCD30 and MV-CD30. Replication kinetics of both viruses did not differ from those of their parental viruses (Figure 1C). Notably, VSV-CD30 and VSV-MV replicated faster and to higher titers than their MV-based counterparts.

\section{Receptor tropism of the CD30-targeted viruses}

Usage of CD30 as entry receptor by the generated CD30-targeted viruses was analyzed on a panel of CHO cells stably expressing either the natural MV receptors CD46 or SLAM, or the target receptor CD30. Parental $\mathrm{CHO}-\mathrm{K} 1$ cells that do not express any of the receptors were not infected, neither by the CD30-targeted viruses, nor their parental viruses (Figure 2A). While MV and 
VSV-MV infected CD46-positive and SLAM-positive cells, both CD30-targeted viruses exclusively infected CHO cells expressing CD30, thus indicating successful retargeting (Figure 2A). The selectivity of the CD30targeted viruses for CD30-positive cells was further verified in a mixed cell culture composed of CD30negative HT1080 and HT1080-CD30 cells. For better discrimination of the two cell types, CD30-negative HT1080-cells stably expressed the red fluorescent protein RFP (HT1080-RFP). Upon infection with the GFP encoding viruses these cells were expected to emit yellow fluorescence. Indeed, infection with MV or VSVMV led to yellow fluorescence, mainly emitted from large syncytia that had formed between both cell types (Figure

A

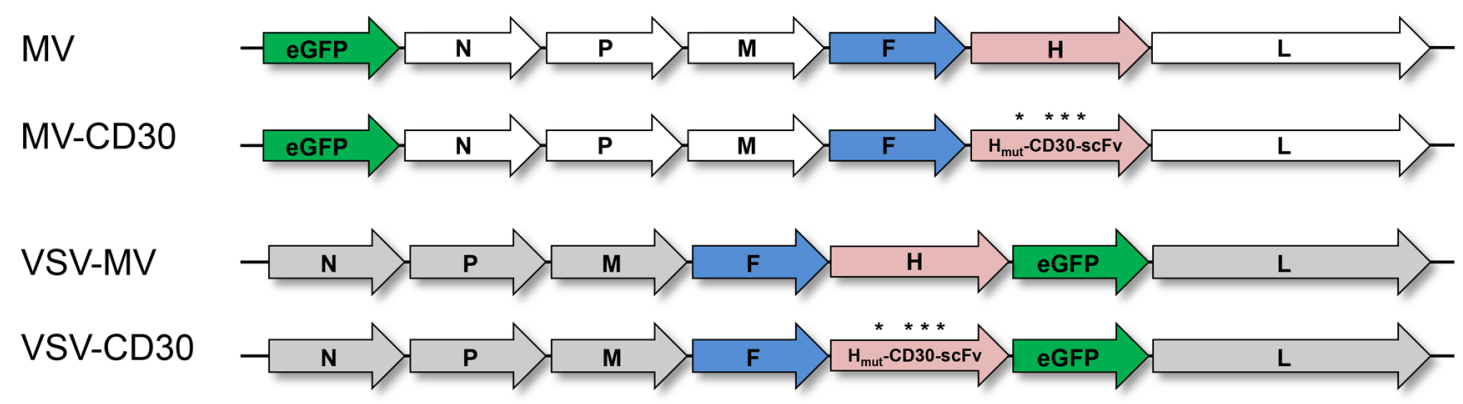

B
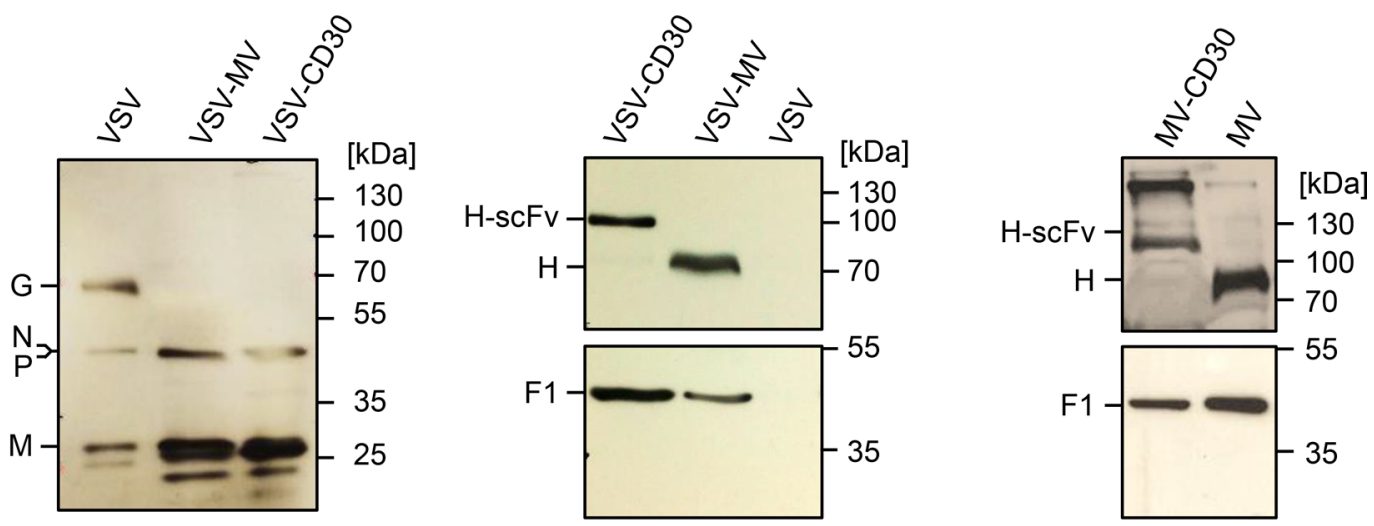

C
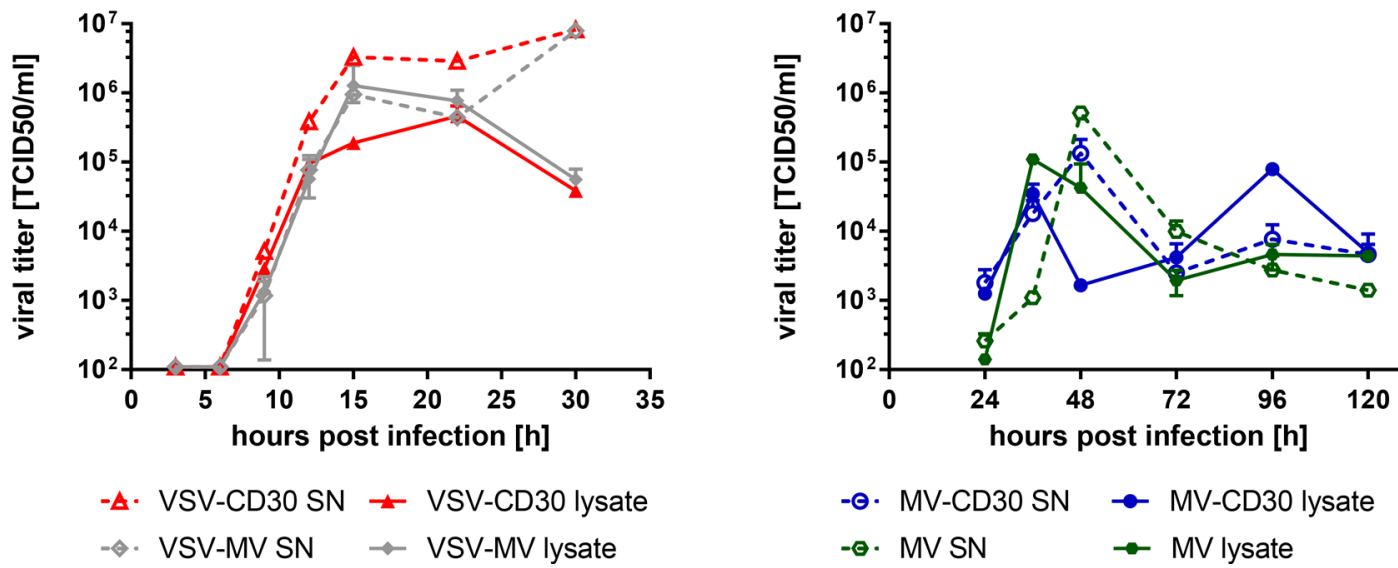

Figure 1: Generation of MV-CD30 and VSV-CD30. (A) Schematic genome organization of the applied oncolytic viruses. Asterisks indicate the mutated residues in $\mathrm{H}$ protein to achieve blinding for the natural MV receptors CD46 and SLAM. The coding sequence for the CD30-specific scFv together with a C-terminal hexa-His-tag (H6) is fused to $\mathrm{H}_{\text {mut }}$. In VSV-CD30 the glycoprotein (G) reading frame was replaced with those of MV-F and $\mathrm{H}_{\text {mut }}-\mathrm{CD} 30 \mathrm{scFv}(\mathbf{B})$ Immunoblot of virus stocks using rabbit- $\alpha$-VSV serum (left panel) or antibodies recognizing the cytoplasmic tail of MV-H (center and right panel top row) or MV-F (center and right panel bottom row). (C) Multi-step growth curves of CD30-targeted viruses and untargeted parental viruses on Vero- $\alpha$ His cells of cell-associated (lysate) and supernatant (SN) virus after infection at a multiplicity of infection (MOI) of 0.3 , respectively. Titers were determined as $50 \%$ tissue culture infective dose (TCID50). $n=2$, error bars: mean \pm SD. 
2B). In sharp contrast, addition of MV-CD30 or VSVCD30 to the co-culture resulted in green fluorescence emitting syncytia, while the red fluorescent cells did not turn yellow nor formed syncytia (Figure 2B). The data demonstrate that the CD30-targeted viruses selectively infect CD30-positive cells, even when these are in direct contact with CD30-negative cells. To finally prove that CD30 was used as entry receptor by VSV-CD30, we assessed competition of infection by soluble CD30. For this purpose, CD30-Fc, a fusion protein composed of the extracellular part of CD30 and the Fc-tag, was expressed and purified as described previously [16] and then preincubated with VSV-CD30 or VSV-MV before infection of HT1080-CD30 cells. The infectivity of VSV-CD30 decreased in a dose dependent manner, while that of VSVMV remained unaffected (Figure 2C).

\section{A}

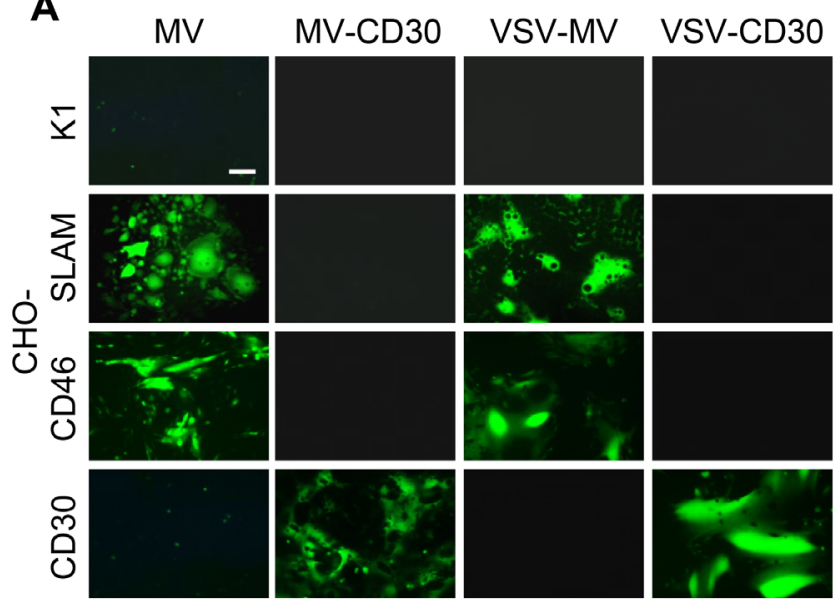

\section{Susceptibility of human classical Hodgkin lymphoma cell lines}

Due to the unavailability of primary HRS-cell cultures cHL patient-derived continuous cell lines were used. These covered two different subtypes of HL, nodular sclerosis (L-428) and mixed cellularity (KM-H2, L-1236) and represented long-standing adequate models for HRS cell physiology with tumorigenic potential [18-20]. Expression levels of the target receptor CD30 and the natural MV receptors CD46 and SLAM were determined by flow cytometry. Flow cytometry analysis confirmed that L-428 and KM-H2 cells expressed CD30 as well as CD46 in a similar high amount, but hardly any SLAM (Figure 3A). Less than half of the L-1236 cells expressed CD30, which were in contrast to the other cell lines not

\section{B}

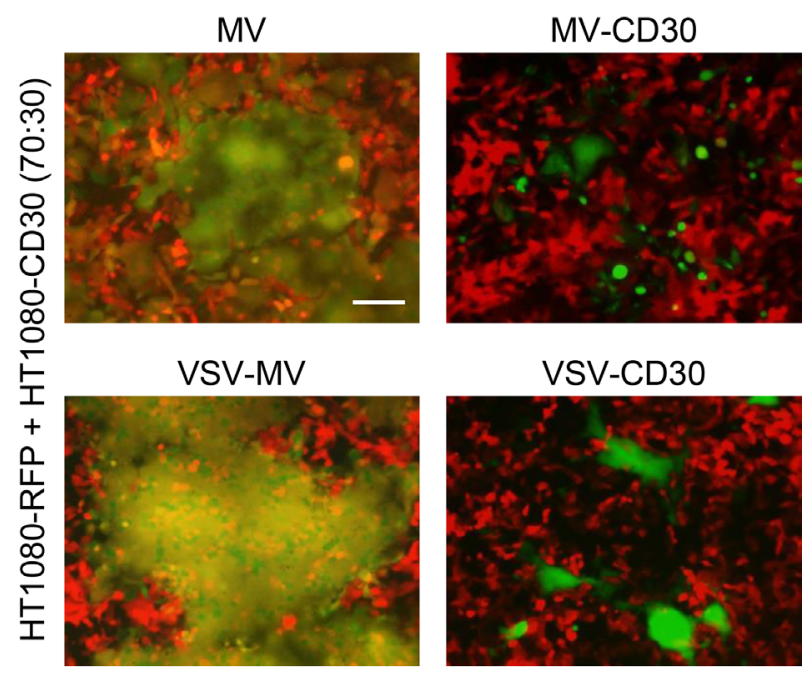

C

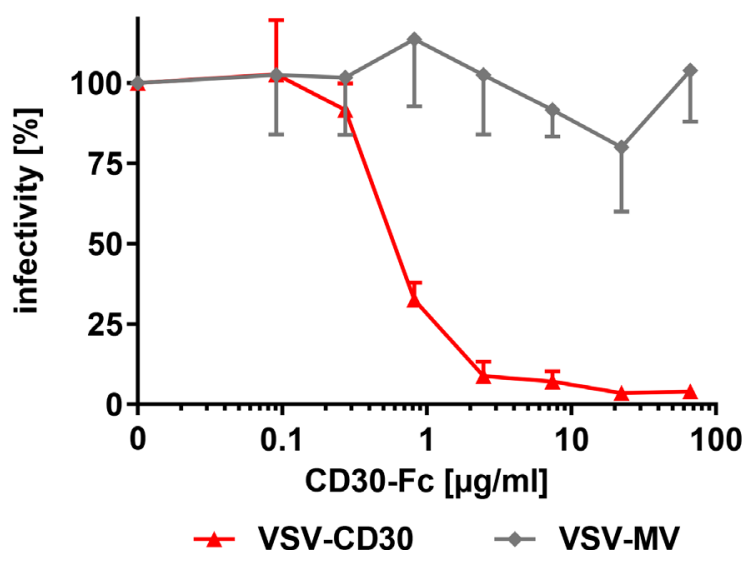

Figure 2: Receptor usage of MV-CD30 and VSV-CD30. (A) CHO-cells stably expressing SLAM, CD46 or CD30 were infected with CD30-targeted viruses or untargeted parental viruses at an MOI of 1 and analyzed by fluorescence microscopy $24 \mathrm{~h}$ (VSV-MV, VSVCD30) or $72 \mathrm{~h}$ (MV, MV-CD30) post infection, respectively. Scale bar $=200 \mu \mathrm{m}$. (B) HT1080-RFP and HT1080-CD30 were co-cultured at a ratio of 70:30 and infected with CD30-targeted viruses or untargeted parental viruses at an MOI of 1 and analyzed by fluorescence microscopy $24 \mathrm{~h}$ (VSV-MV, VSV-CD30) or $72 \mathrm{~h}$ (MV, MV-CD30) post infection, respectively. An overlay of red and green fluorescence is shown. Scale bar $=200 \mu \mathrm{m}$. (C) VSV-MV and VSV-CD30 were pre-incubated for 30 min with increasing amounts of CD30-Fc protein. Subsequently, HT1080-CD30 cells were inoculated and the percentages of infected cells were determined $72 \mathrm{~h}$ later. Relative infectivities were calculated by normalizing the values to mock treated controls. $n=3$, error bars: mean \pm SD. 
only CD46-positive but also SLAM-positive (Figure 3A). Nevertheless, both CD30-targeted viruses as well as their untargeted parental viruses readily infected all three $\mathrm{cHL}$ cell lines and induced the formation of large syncytia (Figure 3B). Finally, the cytotoxic effects exerted by MVCD30 and VSV-CD30 on KM-H2 cells were determined. While within 48 h MV-CD30 did not yet kill cells, VSVCD30 reduced the cell viability to $60 \%$ after $24 \mathrm{~h}$ and to $48 \%$ after $48 \mathrm{~h}$ (Figure 3C). Thus, VSV-CD30 was more potent in killing KM-H2 cells than MV-CD30 in vitro.

\section{Antitumoral activity of VSV-CD30 and MV- CD30 in vivo}

Next, the antitumoral activities of VSV-CD30 and MV-CD30 were evaluated in the KM-H2 xenograft mouse model. KM-H2 cells were implanted subcutaneously into NSG mice. Once tumor had reached a volume of $50 \mathrm{~mm}^{3}$, three intratumoral (i.t.) administrations of in total $3 \times 10^{6}$ TCID50 (MV-CD30 or VSV-CD30) or $3 \times 10^{8}$ TCID50 (VSV-CD30) were performed within one week. Tumors of mice treated with MV-CD30 were only slightly reduced in growth compared to mock treated animals. In contrast,
VSV-CD30 injected tumors were completely blocked in growth for several weeks (Figure 4A-4B). This held true not only for the high dose but also for the low dose, which was equivalent to that of MV-CD30. The oncolytic activity resulted in a significantly prolonged survival of tumorbearing mice treated with VSV-CD30, which was further increased upon the high dose administration (Figure 4C).

Next, the oncolytic potential of VSV-CD30 was assessed after systemic application in the s.c. KM-H2 xenograft mouse model. For this purpose, the animals received three intravenous (i.v.) injections of in total $3 \times 10^{8}$ TCID50 VSV-CD30 or OptiMEM as control within three weeks. Remarkably, also after systemic application VSV-CD30 controlled tumor growth for several weeks and thus equally well as after intratumoral administration (Figure 5A-5B). Quantification of the area under the curve (AUC) revealed that the difference in tumor growth between VSV-CD30 and mock treated was highly significant. On the day the last mock mouse had to be sacrificed all VSV-CD30 treated animals were still alive indicating a clear survival benefit. Spots of infected cells were detected throughout the tumor tissue; equally well in mice that had been injected with VSV-CD30 i.t. as in
A

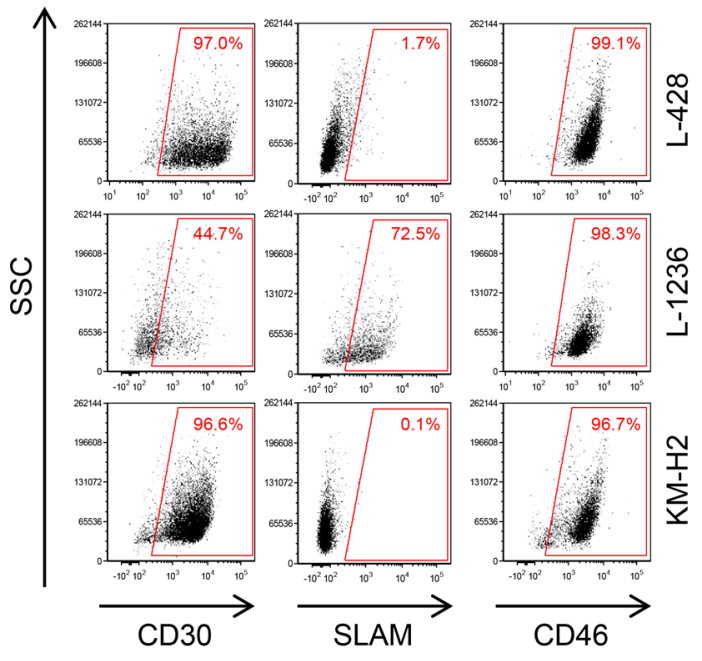

B
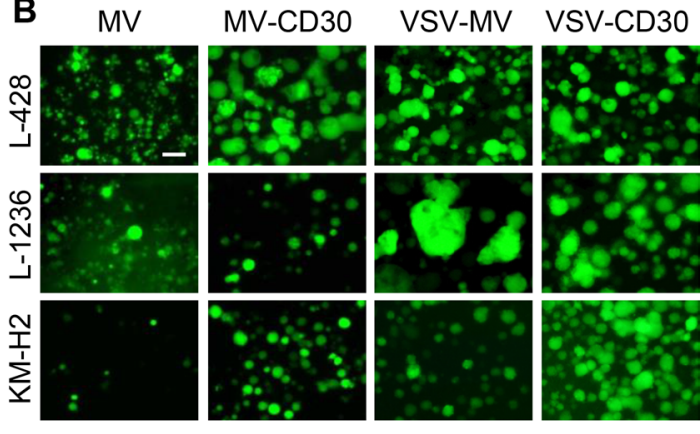

C

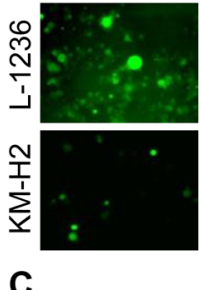

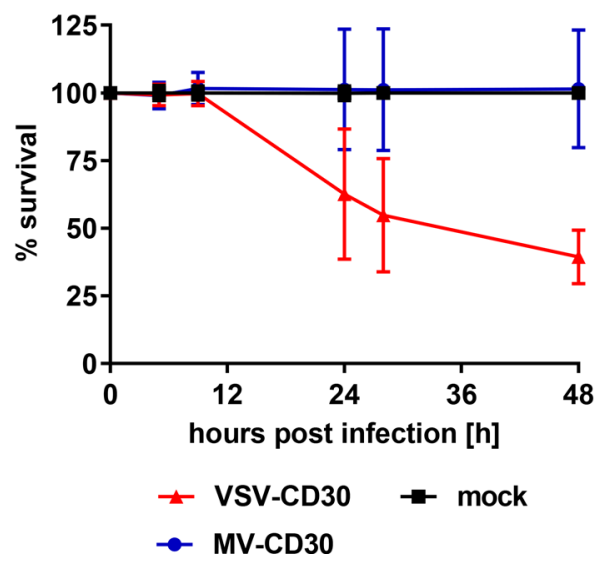

Figure 3: Infection of human classical Hodgkin lymphoma cell lines. (A) FACS analysis of the human cHL cell lines L-428, L-1236 and KM-H2 for expression of CD30, SLAM and CD46. (B) The cHL cell lines L-428, L-1236 and KM-H2 were infected with the CD30-targeted or the untargeted parental viruses at an MOI of 1, respectively, and analyzed by fluorescence microscopy $24 \mathrm{~h}$ (VSVMV, VSV-CD30) or $72 \mathrm{~h}(\mathrm{MV}, \mathrm{MV}-\mathrm{CD} 30)$ post infection. Scale bar $=200 \mu \mathrm{m}$. (C) Cell viability was determined using the RealTime-Glo MT Cell Viability Assay. Human KM-H2 cells were infected with MV-CD30 or VSV-CD30 an MOI of 1, respectively, and viability was determined until $48 \mathrm{~h}$ post infection. $n=3$, Error bars: mean $\pm \mathrm{SD}$. 
i.v. injected mice (Supplementary Figure 1). Notably, there were no signs for any side-effects including neurotoxic effects induced by the oncolytic viruses, neither after intratumoral nor after systemic administration.

Finally, we injected KM-H2-luc cells, which were labeled with the luciferase gene to monitor tumor growth, intravenously into NSG mice followed by VSV-CD30 injection. Luciferase signal intensity of KM-H2-luc cells was monitored and quantified over time for each treatment group (Supplementary Figure 2). At the last day of observation, in all mock-treated animals a high luciferase signal derived from the KM-H2-luc tumor burden was detected. In contrast, five out of eight VSV-CD30 treated animals exhibited considerably decreased luciferase activities (Figure 5C). This indicates that VSV-CD30 is oncolytically active also in a multifocal tumor model.
A

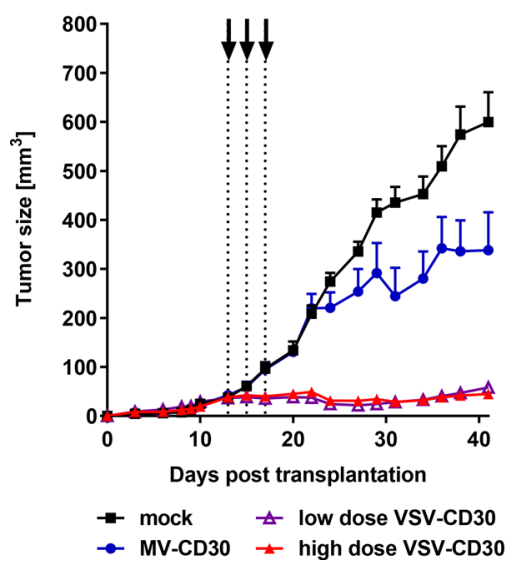

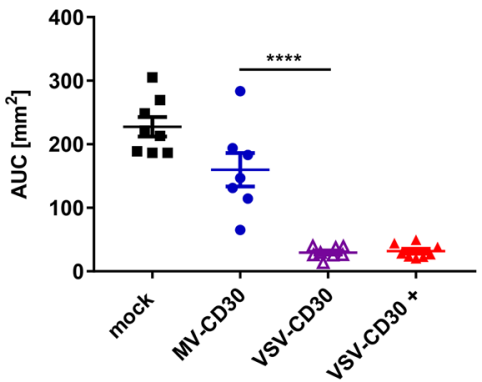

C

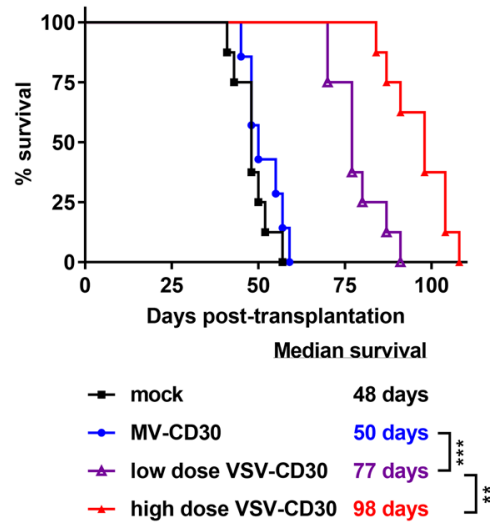

Figure 4: Oncolytic activity in vivo after intratumoral administration. KM-H2 cells were implanted subcutaneously into NSG mice. 13 days post cell implantation, mice received three intratumoral injections over a period of five days (dotted lines) covering a total dose of $3 \times 10^{6}$ TCID50 (MV-CD30, VSV-CD30 low dose) or $3 \times 10^{8}$ TCID50 (VSV-CD30 high dose). (A) Tumor growth curves of MVCD30 (blue circles), VSV-CD30 low dose (purple triangles), VSV-CD30 high dose (red triangles, + ) or mock (black rectangles) treated mice. (B) Calculated area under the curve (AUC) values for tumor growth data shown in (A). One-way ANOVA test (Multiple comparisons), ${ }^{* * * *} p<0.0001$. (C) Kaplan-Meier plot survival analysis. Logrank test (Bonferroni adjusted), ${ }^{* * *} p<0.001,{ }^{* *} p<0.01$. Measles virus (MV)$\mathrm{CD} 30, n=7$; vesicular stomatitis virus (VSV)-CD30 low dose, $n=8$; VSV-CD30 high dose, $n=8$; mock, $n=8$. Error bars: mean \pm SEM.

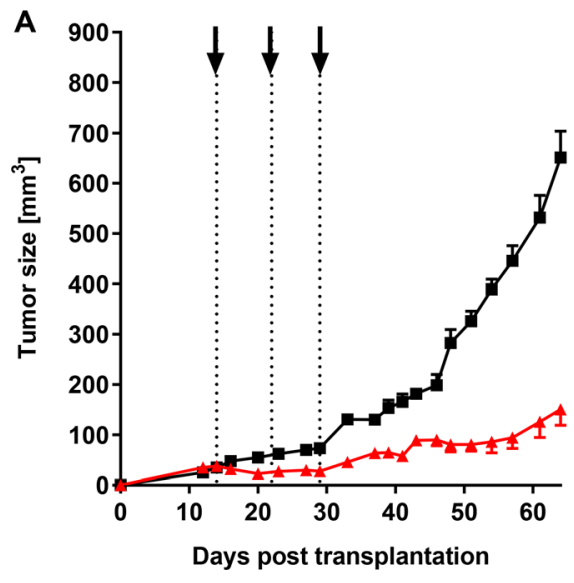

B

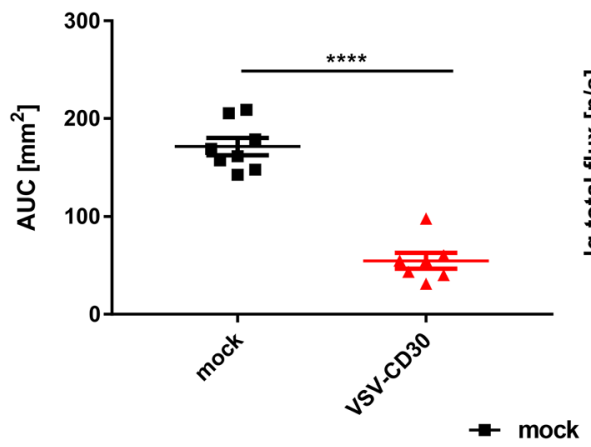

\pm VSV-CD30

Figure 5: Oncolytic activity in vivo after systemic administration of VSV-CD30. (A) KM-H2 cells were implanted subcutaneously into NSG mice. 14 days post cell implantation, a total dose of $3 \times 10^{8}$ TCID50 of VSV-CD30 split in three aliquots was systemically injected weekly (dotted lines). Tumor growth curves of VSV-CD30 (red triangles) or mock (black rectangles) treated mice are shown. (B) Calculated area under the curve (AUC) values for tumor growth data shown in (A). Unpaired $t$ test, ${ }^{* * * *} p<0.0001$. Vesicular stomatitis virus (VSV)-CD30, $n=7$; mock, $n=8$. Error bars: mean \pm SEM. (C) KM-H2-luc cells were injected intravenously into NSG mice. Based on the luciferase signal intensity on day 14 , mice received three intravenous injections of VSV-CD30 covering a total dose of $3 \times 10^{8}$ TCID50 on day 18, 21 and 28 post cell administration. Quantified luciferase signals on day 46 are shown as logarithm of the total flux (p/sec). Unpaired $T$ test, $p=0.07$. VSV-CD30, $n=8$; mock, $n=6$. 


\section{DISCUSSION}

Here, we proved the generation of oncolytic viruses using CD30 as entry receptor. CD30 is a diagnostic marker for several lymphomas including $\mathrm{cHL}$ where it is uniformly expressed in the HRS cells [21]. Belonging to the superfamily of tumor necrosis factor (TNF) receptors and as typical type-1 transmembrane protein, CD30 encompasses a large elongated extracellular part and a cytoplasmic domain that mediates signaling upon binding of the TNF family member CD30L. Expression of CD30 is mainly confined to pathological conditions, which include besides $\mathrm{cHL}$ also activated $\mathrm{T}$ lymphocytes as they occur during virus infection or autoimmune diseases [22]. Activated $\mathrm{T}$ cells could therefore form a potential target for VSV-CD30 or MV-CD30.

In healthy cells, including $\mathrm{T}$ lymphocytes, however, viral infection induces antiviral mechanisms of the innate immune system for example the expression of type-I interferons [23]. Tumor cells, in contrast, accumulate defects in the innate immune system to escape the immune response. These defects render them sensitive towards infection especially with RNA viruses [24]. Indeed, primary human $\mathrm{T}$ cells turned out to be completely protected from infection with VSV-CD30 or VSV-MV, despite the presence of $\mathrm{CD} 30$ on the cell surface and several days of cell cultivation after incubation with high MOI (Supplementary Figure 3). In contrast, some infected cells and syncytia were detectable with MV and to a minor extent also with MV-CD30 (Supplementary Figure 3). Thus, CD30 targeting does not extend the tropism of VSV or MV to T lymphocytes. This is well in line with the previous observation that targeting these viruses to CD133 does not lead to productive infection of hematopoietic stem cells, most likely due to the high sensitivity against interferon [15]. Taking in addition the proven safety of high dose systemic administration of MV in multiple myeloma patients into account [25] we expect that targeting CD30 results in safe products exerting, if at all, only subtle side-effects.

Our approach of CD30-targeting relied on engineered MV envelope glycoproteins displaying the stability-engineered CD30-specific scFv HRS3opt2\#2. This $\mathrm{scFv}$ was proven to be highly stable and specific in mediating gene delivery by lentiviral vectors pseudotyped with these MV glycoproteins [26]. By replacing the envelope genes in the genomes of MV and VSV with those encoding the CD30-targeted glycoproteins, MV-CD30 and VSV-CD30 were generated. Both viruses exhibited a restricted infection and efficient spreading within $\mathrm{CD} 30^{+}$positive cHL cells. When comparing their oncolytic activities VSV-CD30 turned out to be more efficient than MV-CD30. This is in line with previously published data on chimeric VSV-MV viruses targeted to Her2/neu or CD133 [15, 27]. VSV-CD30 thus is now the third example of a receptor-targeted VSV. Although there is an obvious tendency for VSV to be oncolytically more effective than MV this cannot be generalized and has to be evaluated for each type of tumor separately. Especially the strong sensitivity towards interferon might be disadvantageous for VSV on certain tumor types as recently shown for VSV-CD133 and glioma tumor spheres, which turned out to be more susceptible to the MV counterpart MV-CD133 [15].

For the three HL cell lines used in this study, there were no hints for any impairment of the oncolytic activity by interferons detectable suggesting that cHL may be well compatible with the VSV-mediated oncolytic activity. Especially in vivo, where tumor cell lines often become responsive to interferons [28], VSV-CD30 was highly active and prevented outgrowth of the subcutaneously injected cHL cells. Notably, this was the case not only after local intratumoral but also after systemic intravenous injection, again underscoring the high activity of VSVCD30. Primary HRS cells are basically not recoverable from single cell suspensions of HL-affected lymph nodes or other biopsy specimen of HL patients. Accordingly, only few HL cell lines were established during the last decades. The HL cell lines assessed in our study were frequently used for research purpose in important studies $[18,19]$. They display three independent patient cases, as they were derived from pleural effusions (KM-H2, L-428) or peripheral blood (L-1236), and cover two different subtypes of HL, namely nodular sclerosis (L-428) and mixed cellularity (KM-H2, L-1236). A preclinical model reflecting the low abundance of tumor cells within the affected tissue is therefore so far not available [29, 30]. Hence, we have assessed VSV-CD30 in a mouse model of disseminated growing cHL tumor cells and found a substantial reduction in tumor growth at least for some of the mice. This illustrates nicely the power of CD30 targeting and suggests that VSV-CD30 may also effectively access and kill cHL tumor cells in patients and thus become a novel therapeutic option for cHL.

An interesting property of $\mathrm{cHL}$ that was recently discovered refers to the presence of $\mathrm{CD} 30^{+}$-positive extracellular vesicles (CD30-EVs) shed from cHL tumor cells [31]. Interestingly, the authors found that CD30EVs positively contribute to the clinical efficacy of brentuximab vedotin. They demonstrated that $\mathrm{CD} 30-\mathrm{EV}$ s migrate to $\mathrm{CD} 30$-negative but $\mathrm{CD} 30$ ligand $(\mathrm{CD} 30 \mathrm{~L})$ positive bystander cells in the tumor environment, mainly consisting of mast cells and eosinophils. Brentuximab vedotin binds to CD30-EVs, is then carried to the bystander cells where it becomes internalized and cytotoxically active. Since the CD30-targeted viruses described here bind to CD30 similarly efficient and selective as antibodies, it can be expected that it will be transported via CD30-EVs as well. If this leads to infection and killing of the bystander cells in the tumor microenvironment will be an interesting issue to be further investigated. 
Besides cHL also other types of cancer such as anaplastic large cell lymphoma (ALCL), cutaneous CD30positive lymphoproliferative disorders, lymphomatoid papulomatosis, diffuse large B cell lymphoma or adult $\mathrm{T}$ cell leukemia can be CD30 positive $[6,21]$. Beyond that, also solid tumors especially mesothelioma and germ cell tumors can express CD30 [32]. The data we present here for the oncolytic activity of VSV-CD30 warrant further testing of this virus not only for applications in cHL, but also in these other CD30-positive disorders. Before clinical trials can be envisaged preclinical testing of VSV-CD30 will have to be extended assessing the oncolytic activity on $\mathrm{cHL}$ patient biopsy material and to toxicity testing. The latter will have to focus especially on neurotoxicity which is a concern with VSV-derived oncolytic viruses [33]. Envelope modification as done here, however, has often resulted in attenuation of VSV [34]. Upon completion, VSV-CD30 will add to a growing list of CD30-targeted therapeutics that include besides antibodies also CD30-specific AAV vectors [35] and CAR T cells $[30,36]$. Combining VSV-CD30 with these or other cancer therapeutics such as checkpoint inhibitors will be another future option to be explored.

\section{MATERIALS AND METHODS}

\section{Cells}

BHK-21 (ATCC CCL-10), HEK 293-T (ATCC CRL-11268) and CHO-K1 (ATCC CCL-61) cells were cultured in DMEM (Sigma-Aldrich, Germany) supplemented with 10\% FCS (Biochrom, Germany) and $2 \mathrm{mM}$ L-glutamine (Sigma-Aldrich, Germany). Generation and cultivation of Vero- $\alpha$ His [17], CHOCD46 [37] and CHO-SLAM [38] has been described earlier. CHO-CD30 were derived from CHO-K1 cells (ATCC CCL-61) by stable expression of CD30 which was achieved upon selection with $10 \mu \mathrm{g} / \mathrm{ml}$ puromycin. HT1080-CD30 [16] and HT1080-RFP cells [39] have been described. The human cHL cell lines KM-H2, L-428 and L-1236 were obtained from the German Collection of Microorganisms and Cell Cultures, Germany and cultured in RPMI 1640 (Biowest, France) supplemented with $10 \%$ FCS and 2 mM L-glutamine. KM-H2-luc cells were derived from KM-H2 cells by stable expression of the luciferase gene which was achieved upon selection with $10 \mu \mathrm{g} / \mathrm{ml}$ puromycin.

\section{Cloning and rescue of MV-CD30 and VSV-CD30}

As targeting ligand for CD30 we used the stability optimized scFv HRS3opt2\#2 [16]. For cloning of the genome plasmid of MV-CD30 the sequence for retargeted $\mathrm{H}$ was cut out of the expression plasmid $\left(\mathrm{pCG}^{-\mathrm{H}_{\text {mut }}}\right.$ scFvCD30opt2.2-6His) [16] using PacI/SpeI restriction sites. PacI/SpeI flanking insert was transferred into the
CMV-promotor driven $\mathrm{MV}_{\mathrm{NSe}}$ genome plasmid $\mathrm{p}(+)$

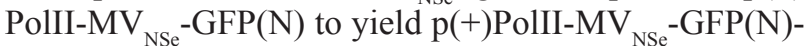
$\alpha C D 30 o p t 2.2$ (pMV-CD30). For rescue of MV-CD30 the PolII rescue system [40] was used.

For cloning of the genome plasmid of VSV-CD30 the sequence for retargeted $\mathrm{H}$ was cut out of the expression plasmid (pCG- $\mathrm{H}_{\text {mut }}$-scFvCD30opt2.2-6His) using SfiI/ NotI restriction sites. The backbone plasmid for VSV-MV chimera (pMC11-VSVFH-eGFP) was described earlier where the VSV-G glycoprotein $(1.6 \mathrm{~kb})$ at position 4 of the full-length VSV genome was replaced by the MV-F $(1.8 \mathrm{~kb})$ and $\mathrm{MV}-\mathrm{H}(2 \mathrm{~kb})$ at positions 4 and 5 [14]. SfiI/ NotI flanking insert was transferred into the T7-promotor driven VSV-MV genome plasmid pMC11-VSVFH-eGFP to yield pMC11-VSVFH- $\alpha$ CD30opt2.2 (pVSV-CD30).

VSV-CD30 was rescued as described previously for VSV-CD133, essentially by making use of the helper plasmids coding for VSV-N, -P and -L, BHK-21 cells, a complementing VSV-G encoding plasmid and the T7 RNA polymerase provided by a modified vaccinia virus Ankara (MVA-T7-Pol) [15]. The rescued chimeric virus was purified by single syncytia isolation. All viruses were grown and titrated on Vero- $\alpha$ His cells to calculate the $50 \%$ tissue culture infective dose (TCID50/ml).

\section{Virus growth kinetics}

$2 \times 10^{5}$ Vero- $\alpha$ His cells were seeded in a 12 -well plate. Cells were infected with an MOI of 0.03. Samples were harvested every $24 \mathrm{~h}$. For this purpose, virus was collected from the supernatant by centrifugation and stored at $-80^{\circ} \mathrm{C}$ for further analysis. For harvesting cellassociated virus, adherent cells were scraped into $1 \mathrm{ml}$ OptiMEM (Thermo Fisher Scientific, USA) and lysed by freeze-thawing in liquid nitrogen. Cell debris was removed by centrifugation. Resulting cell-associated virus containing medium was stored at $-80^{\circ} \mathrm{C}$. Titers were determined as TCID50/ml on Vero- $\alpha$ His cells.

\section{Immunoblotting}

Immunoblotting was performed as described previously [15]. Membranes were incubated with rabbit sera recognizing MV-F (Abcam, Great Britain), MV-H (Abcam, Great Britain) for detection of MV-CD30 and $\mathrm{MV}$, the cytoplasmic tail of MV-H [39] for detection of VSV-CD30, VSV-MV, and VSV, or rabbit- $\alpha$-VSV serum as described [41].

\section{Flow cytometry analysis}

Expression of human CD30 was detected by a phycoerythrin (PE)-labeled mouse CD30 antibody (clone: Ki-2, Miltenyi Biotech, Germany). Human CD46 was detected by a fluorescein isothiocyanate (FITC)-labeled mouse CD46 antibody (clone: MEM-258, BioLegend, 
USA). Human CD150 (SLAM) was detected by a PElabeled mouse CD150 (SLAM) antibody (clone: A12 (7D4), BioLegend, USA). Viability of cells was analyzed using the LIVE/DEAD Fixable Violet Dead Cell Stain Kit (Molecular Probes, USA). Flow cytometry analysis was performed using the MACSQuant Analyzer 10 (Miltenyi Biotec, Germany) and data were analyzed using FCS Express version 4.

\section{Cytotoxicity assay}

$1 \times 10^{5} \mathrm{KM}-\mathrm{H} 2$ cells were seeded in a 96-well and infected with VSV-CD30 or MV-CD30 at an MOI of 1. Cell viability was analyzed using the RealTime-Glo MT Cell Viability Assay (Promega, Germany) according to the manufacturer's protocol.

\section{Immunofluorescence staining of cryo sections}

Cryo-sections of tumor tissue were performed as described previously [15]. For immunofluorescence staining against CD31 slices were incubated with the rat anti-GFP antibody (Dianova, Germany) overnight at $4^{\circ} \mathrm{C}$, followed by incubation with the donkey anti-rat Alexa647-coupled secondary antibody (Jackson ImmunoResearch, USA).

\section{Animal experiments}

All animal experiments were carried out in compliance with the regulations of the German animal protection law. To analyze the antitumoral effect of oncolytic viruses in the s.c. HL xenograft model, $1 \times 10^{7}$ $\mathrm{KM}-\mathrm{H} 2$ cells in $50 \mu \mathrm{l}$ PBS were mixed with $50 \mu \mathrm{l}$ Matrigel Basement Membrane Matrix (Corning, USA) and implanted into the flank of 6-8 weeks old female NSG mice (Charles River, Germany). Tumor growth was monitored regularly using a digital caliper. When tumors became palpable (at an average size of $50 \mathrm{~mm}^{3}$ ), mice were randomized into groups. They received in total three intratumoral injections of oncolytic virus. MV-CD30 (1 x 10 ${ }^{6}$ TCID50 per injection) or VSV-CD30 (low dose: 1 x $10^{6}$ TCID50, high dose: 1 × $10^{8}$ TCID50 per injection) were administered in $50 \mu$ l OptiMEM every second day. Mock control animals received intratumoral injections of $50 \mu \mathrm{l}$ OptiMEM (Thermo Fisher Scientific, USA). For systemic application, $1 \times 10^{8}$ TCID50 VSVCD30 in $200 \mu \mathrm{l}$ OptiMEM or OptiMEM only as mock control were injected into the tail vein. In total, mice received three injections, with one injection per week. Differences in tumor growth were afterwards quantified via determination of area under the curve (AUC) [42].

For the multifocal tumor model, $2 \times 10^{6} \mathrm{KM}-\mathrm{H} 2-\mathrm{luc}$ cells stably expressing the luciferase gene, were injected in $200 \mu \mathrm{l}$ PBS intravenously via the tail vein into 6-8 weeks old female NSG mice. To follow up tumor progression, luciferase signals were detected by in vivo Imaging (IVIS
Spectrum; Perkin Elmer, Germany) after intraperitoneal injection of $150 \mu \mathrm{g}$ D-luciferin (Perkin Elmer, Germany) per gram body weight. Imaging data were obtained $10 \mathrm{~min}$ after luciferin injection. 14 days post cell administration animals were separated into treatment groups according to the mean luciferase signal intensity calculated via the Living Image Software (Caliper Life Sciences). Mice received three intravenous injections via the tail vein of VSV-CD30 ( $1 \times 10^{8}$ TCID50 per dose) in $200 \mu$ or PBS as mock control within 10 days. Mice were euthanized when the tumor had reached a size of $>800 \mathrm{~mm}^{3}$ or when more than $20 \%$ of their body weight was lost.

\section{Author contributions}

J.D.S.H. designed and performed experiments and contributed to writing of the manuscript. D.K. performed experiments. B.R., S.N. and M.L.H. contributed protocols and reagents and evaluated data. J.R., A.P., T.F., I.C.S. contributed unique reagents. C.J.B. acquired grants, supervised work and wrote the manuscript. A.M. conceived the study, acquired grants, supervised work and contributed to writing of the manuscript.

\section{ACKNOWLEDGMENTS}

The authors like to thank Tatjana Weidner for excellent technical assistance, Kay-Martin Hanschmann for help with statistics as well as Kah-Whye Peng and Camilo Ayala-Breton for kindly providing the VSVMV chimera backbone plasmid pMC11-VSVFH-eGFP. This work was supported by grants of the LOEWE Center for Cell and Gene Therapy Frankfurt funded by Hessisches Ministerium für Wissenschaft und Kunst (III L 4-518/17.004 [2010]) to A.M., S.N. and C.J.B.

\section{CONFLICTS OF INTEREST}

None.

\section{REFERENCES}

1. Küppers R. The biology of Hodgkin's lymphoma. Nat Rev Cancer. 2009; 9:15-27.

2. Pileri SA, Ascani S, Leoncini L, Sabattini E, Zinzani PL, Piccaluga PP, Pileri A, Giunti M, Falini B, Bolis GB, Stein H. Hodgkin's lymphoma: the pathologist's viewpoint. J Clin Pathol. 2002; 55:162-176.

3. Kelley TW, Pohlman B, Elson P, Hsi ED. The ratio of FOXP3 + regulatory $\mathrm{T}$ cells to granzyme $\mathrm{B}+$ cytotoxic $\mathrm{T} /$ NK cells predicts prognosis in classical Hodgkin lymphoma and is independent of bcl-2 and MAL expression. Am J Clin Pathol. 2007; 128:958-965.

4. Blank O, von Tresckow B, Monsef I, Specht L, Engert A, Skoetz N. Chemotherapy alone versus chemotherapy plus 
radiotherapy for adults with early stage Hodgkin lymphoma. Cochrane Database Syst Rev. 2017; 4:CD007110.

5. Kasamon YL, de Claro RA, Wang Y, Shen YL, Farrell AT, Pazdur R. FDA Approval Summary. Nivolumab for the Treatment of Relapsed or Progressive Classical Hodgkin Lymphoma. Oncologist. 2017; 22:585-591.

6. Scott LJ. Brentuximab Vedotin. A Review in CD30-Positive Hodgkin Lymphoma. Drugs. 2017; 77:435-445.

7. Chen R, Gopal AK, Smith SE, Ansell SM, Rosenblatt JD, Savage KJ, Connors JM, Engert A, Larsen EK, Huebner D, Fong A, Younes A. Five-year survival and durability results of brentuximab vedotin in patients with relapsed or refractory Hodgkin lymphoma. Blood. 2016; 128:1562-1566.

8. Breitbach CJ, Lichty BD, Bell JC. Oncolytic Viruses: Therapeutics With an Identity Crisis. EBioMedicine. 2016; 9:31-36.

9. Pol J, Kroemer G, Galluzzi L. First oncolytic virus approved for melanoma immunotherapy. Oncoimmunology. 2016; 5:e1115641.

10. Campadelli-Fiume G, Petrovic B, Leoni V, Gianni T, Avitabile E, Casiraghi C, Gatta V. Retargeting Strategies for Oncolytic Herpes Simplex Viruses. Viruses. 2016; 8:63.

11. Msaouel P, Iankov ID, Allen C, Russell SJ, Galanis E. Oncolytic measles virus retargeting by ligand display. Methods Mol. Biol. 2012; 797:141-162.

12. Msaouel P, Opyrchal M, Dispenzieri A, Peng KW, Federspiel MJ, Russell SJ, Galanis E. Clinical Trials with Oncolytic Measles Virus: Current Status and Future Prospects. Curr Cancer Drug Targets. 2017 Feb 22. [Epub ahead of print].

13. Aref S, Bailey K, Fielding A. Measles to the Rescue: A Review of Oncolytic Measles Virus. Viruses. 2016; 8:E294.

14. Ayala-Breton C, Russell LO, Russell SJ, Peng KW. Faster replication and higher expression levels of viral glycoproteins give the vesicular stomatitis virus/measles virus hybrid VSV-FH a growth advantage over measles virus. J Virol. 2014; 88:8332-8339.

15. Kleinlützum D, Hanauer JDS, Muik A, Hanschmann KM, Kays SK, Ayala-Breton C, Peng KW, Mühlebach MD, Abel T, Buchholz CJ. Enhancing the Oncolytic Activity of CD133-Targeted Measles Virus. Receptor Extension or Chimerism with Vesicular Stomatitis Virus Are Most Effective. Front Oncol. 2017; 7:10.

16. Friedel T, Hanisch LJ, Muth A, Honegger A, Abken H, Plückthun A, Buchholz CJ, Schneider IC. Receptor-targeted lentiviral vectors are exceptionally sensitive toward the biophysical properties of the displayed single-chain Fv. Protein Eng Des Sel. 2015; 28:93-106.

17. Nakamura T, Peng KW, Harvey M, Greiner S, Lorimer IA, James CD, Russell SJ. Rescue and propagation of fully retargeted oncolytic measles viruses. Nat Biotech. 2005; 23:209-214.

18. Mathas S, Janz M, Hummel F, Hummel M, Wollert-Wulf B, Lusatis S, Anagnostopoulos I, Lietz A, Sigvardsson M, Jundt F, Jöhrens K, Bommert K, Stein H, et al. Intrinsic inhibition of transcription factor E2A by HLH proteins ABF-1 and Id2 mediates reprogramming of neoplastic B cells in Hodgkin lymphoma. Nat Immunol. 2006; 7:207-215.

19. Steidl C, Shah SP, Woolcock BW, Rui L, Kawahara M, Farinha P, Johnson NA, Zhao Y, Telenius A, Neriah SB, McPherson A, Meissner B, Okoye UC, et al. MHC class II transactivator CIITA is a recurrent gene fusion partner in lymphoid cancers. Nature. 2011; 471:377-381.

20. Tiacci E, Döring C, Brune V, van Noesel CJ, Klapper W, Mechtersheimer G, Falini B, Küppers R, Hansmann ML. Analyzing primary Hodgkin and Reed-Sternberg cells to capture the molecular and cellular pathogenesis of classical Hodgkin lymphoma. Blood. 2012; 120:4609-4620.

21. Pierce JMR, Mehta A. Diagnostic, prognostic and therapeutic role of CD30 in lymphoma. Expert Rev Hematol. 2017; 10:29-37.

22. Horie R, Watanabe T. CD30: expression and function in health and disease. Semin Immunol. 1998; 10:457-70.

23. Katze MG, He Y, Gale M Jr. Viruses and interferon. A fight for supremacy. Nature Reviews Immunology. 2002; 2:nri888.

24. Stojdl DF, Lichty BD, tenOever BR, Paterson JM, Power AT, Knowles S, Marius R, Reynard J, Poliquin L, Atkins H, Brown EG, Durbin RK, Durbin JE, et al. VSV strains with defects in their ability to shutdown innate immunity are potent systemic anti-cancer agents. Cancer Cell. 2003; $4: 263-275$.

25. Dispenzieri A, Tong C, LaPlant B, Lacy MQ, Laumann K, Dingli D, Zhou Y, Federspiel MJ, Gertz MA, Hayman S, Buadi F, O'Connor M, Lowe VJ, et al. Phase I trial of systemic administration of Edmonston strain of measles virus genetically engineered to express the sodium iodide symporter in patients with recurrent or refractory multiple myeloma. Leukemia. 2017; 31:2791-8.

26. Friedel T, Jung-Klawitter S, Sebe A, Schenk F, Modlich U, Ivics Z, Schumann GG, Buchholz CJ, Schneider IC. CD30 Receptor-Targeted Lentiviral Vectors for Human Induced Pluripotent Stem Cell-Specific Gene Modification. Stem Cells and Development. 2016; 25:729-39.

27. Ayala Breton C, Wikan N, Abbuhl A, Smith DR, Russell SJ, Peng KW. Oncolytic potency of HER-2 retargeted VSV-FH hybrid viruses: the role of receptor ligand affinity. Mol Ther Oncolytics. 2015; 2:15012.

28. Liu YP, Suksanpaisan L, Steele MB, Russell SJ, Peng KW. Induction of antiviral genes by the tumor microenvironment confers resistance to virotherapy. Sci Rep. 2013; 3:2375.

29. Rengstl B, Newrzela S, Heinrich T, Weiser C, Thalheimer FB, Schmid F, Warner K, Hartmann S, Schroeder T, Küppers R, Rieger MA, Hansmann ML. Incomplete cytokinesis and re-fusion of small mononucleated Hodgkin cells lead to giant multinucleated Reed-Sternberg cells. Proc Natl Acad Sci U S A. 2013; 110:20729-20734.

30. Rengstl B, Schmid F, Weiser C, Döring C, Heinrich T, Warner K, Becker PSA, Wistinghausen R, Kameh-Var S, 
Werling E, Billmeier A, Seidl C, Hartmann S, et al. Tumorinfiltrating HLA-matched CD4(+) T cells retargeted against Hodgkin and Reed-Sternberg cells. Oncoimmunology. 2016; 5:e1160186.

31. Hansen HP, Trad A, Dams M, Zigrino P, Moss M, Tator M, Schön G, Grenzi PC, Bachurski D, Aquino B, Dürkop $\mathrm{H}$, Reiners KS, von Bergwelt-Baildon $\mathrm{M}$, et al. CD30 on extracellular vesicles from malignant Hodgkin cells supports damaging of CD30 ligand-expressing bystander cells with Brentuximab-Vedotin, in vitro. Oncotarget. 2016; 7:30523-30535. https://doi.org/10.18632/oncotarget.8864.

32. Berger GK, Gee K, Votruba C, McBride A, Anwer F. Potential application and prevalence of the CD30 (Ki1) antigen among solid tumors: A focus review of the literature. Crit Rev Oncol Hematol. 2017; 113:8-17.

33. Hastie E, Grdzelishvili VZ. Vesicular stomatitis virus as a flexible platform for oncolytic virotherapy against cancer. Journal of General Virology. 2012; 93:2529-2545.

34. Muik A, Stubbert LJ, Jahedi RZ, Geibeta Y, Kimpel J, Dold C, Tober R, Volk A, Klein S, Dietrich U, Yadollahi B, Falls $\mathrm{T}$, Miletic $\mathrm{H}$, et al. Re-engineering vesicular stomatitis virus to abrogate neurotoxicity, circumvent humoral immunity, and enhance oncolytic potency. Cancer Res. 2014; 74:3567-3578.

35. Muik A, Reul J, Friedel T, Muth A, Hartmann KP, Schneider IC, Münch RC, Buchholz CJ. Covalent coupling of highaffinity ligands to the surface of viral vector particles by protein trans-splicing mediates cell type-specific gene transfer. Biomaterials. 2017; 144:84-94.

36. Ramos CA, Ballard B, Zhang H, Dakhova O, Gee AP, Mei Z, Bilgi M, Wu MF, Liu H, Grilley B, Bollard CM, Chang BH, Rooney CM, et al. Clinical and immunological responses after CD30-specific chimeric antigen receptor-redirected lymphocytes. J Clin Invest. 2017; 127:3462-3471.
37. Manchester M, Liszewski MK, Atkinson JP, Oldstone MB. Multiple isoforms of CD46 (membrane cofactor protein) serve as receptors for measles virus. Proc Natl Acad Sci U S A. 1994; 91:2161-2165.

38. Tatsuo H, Ono N, Tanaka K, Yanagi Y. SLAM (CDw150) is a cellular receptor for measles virus. Nature. 2000; 406:893-897.

39. Bach P, Abel T, Hoffmann C, Gal Z, Braun G, Voelker I, Ball CR, Johnston IC, Lauer UM, Herold-Mende C, Mühlebach MD, Glimm H, Buchholz CJ. Specific elimination of CD133+ tumor cells with targeted oncolytic measles virus. Cancer Res. 2013; 73:865-874.

40. Martin A, Staeheli P, Schneider U. RNA Polymerase IIControlled Expression of Antigenomic RNA Enhances the Rescue Efficacies of Two Different Members of the Mononegavirales Independently of the Site of Viral Genome Replication. J Virol. 2006; 80:5708-5715.

41. Hoffmann M, Wu YJ, Gerber M, Berger-Rentsch M, Heimrich B, Schwemmle M, Zimmer G. Fusion-active glycoprotein $\mathrm{G}$ mediates the cytotoxicity of vesicular stomatitis virus M mutants lacking host shut-off activity. J Gen Virol. 2010; 91:2782-2793.

42. Duan F, Simeone S, Wu R, Grady J, Mandoiu I, Srivastava PK. Area under the curve as a tool to measure kinetics of tumor growth in experimental animals. J Immunol Methods. $2012 ; 382: 224-228$. 